\title{
The Usefulness of Measuring Anti-Xa Activity for the Prediction of Bleeding Adverse Events During Postsurgical Fondaparinux Anticoagulant Therapy
}

\author{
KOICHIRO NIWA*, KAZUHIRO SAKAMOTO*, HiSASHI RO*, Jun AOKI*, ShinyA MUNAKATA*, \\ SHun ISHIYAMA*, KiICHI SUGIMOTO*, HiROHIKO KAMIYAMA*, MAKOTO TAKAHASHI*, \\ YUTAKA KOJIMA*, Michitoshi GOTO*, YUichI TOMIKI* \\ * Department of Coloproctological Surgery, Juntendo University Faculty of Medicine, Tokyo, Japan
}

\begin{abstract}
Objective: Anticoagulation therapy is commonly used to prevent venous thromboembolism after abdominal surgery. However, bleeding during anticoagulation therapy is a problem, particularly in use of fondaparinux (FPX), for which there is no useful monitoring method. In this study, we examined the usefulness of measurement of the inhibitory activity of activated blood coagulation factor X (anti-Xa activity) for prediction of bleeding.

Subjects and Methods: The subjects were 67 patients who underwent anticoagulation therapy with FPX after surgery for colorectal cancer. FPX (2.5 mg) was subcutaneously administered from Day 1 to Day 5. Measurement of anti-Xa activity were performed on Days 1, 3, 5 and 7 to evaluate the relationship between anti-Xa activity and bleeding.

Results: No symptomatic venous thromboembolism developed in any patient. Bleeding was found in 11 subjects (16.4\%). The anti-Xa activity gradually rose after initiation of administration in both the bleeding and non-bleeding groups until day 5 . When the anti-Xa activity was compared between the bleeding and non-bleeding groups, the anti-Xa activity was higher in the bleeding group on day $3(\mathrm{p}=0.002)$.

Conclusion: Careful attention to bleeding is required in anticoagulant therapy with FPX. Measurement of anti-Xa activity may be useful for prediction of bleeding.
\end{abstract}

Key words: anti-Xa activity, fondaparinux, venous thromboembolism, bleedig, colorectal cancer

\section{Introduction}

A risk of venous thromboembolism (VTE) has been reported after abdominal surgery, and postoperative anticoagulant therapy has recently been introduced to prevent $\mathrm{VTE}^{12}$ 2).

Unfractionated heparin, which has been used for anticoagulant therapy, is a 5 -sugar polymer structure that binds to a specific site on antithrombin III (AT III). Various sugar chains bind to this site, and this interaction inhibits both activated coagulation factor $\mathrm{X}(\mathrm{Xa})$ and thrombin. However, development of a novel anticoagulant is essential because the effect is not stable, the frequency of bleeding is high, and there is a risk of heparin-induced thrombocytopenia ${ }^{3)-5}$. Fondaparinux (FPX) is a chemically synthesized drug comprised of only the 5-sugar structure that is the AT III-binding region, and it selectively inhibits $\mathrm{Xa}^{3}$. The postoperative VTE-preventive effect by FPX was investigated mainly in the orthopedic field where it was found to be useful ${ }^{6)}$ 7). The successful use of FPX after abdominal surgery and for obese patients has also recently been reported ${ }^{8)-10)}$. On the other hand, an increase in the risk of bleeding adverse events (bleeding) was also reported ${ }^{11}$. FPX cannot be

Corresponding author: Koichiro Niwa

Department of Coloproctological Surgery, Juntendo University Faculty of Medicine

2-1-1 Hongo, Bunkyo-ku, Tokyo 113-8421, Japan

TEL: +81-3-3813-3111 FAX: +81-3-3813-0731 E-mail: kniwa@juntendo.ac.jp

[Received May 11, 2016] ‘Accepted June 20,2016〕

Copyright (C) 2016 The Juntendo Medical Society. This is an open access article distributed under the terms of Creative Commons Attribution License (CC BY), which permits unrestricted use, distribution, and reproduction in any medium, provided the original source is properly credited. doi: $10.14789 /$ jmj .62 .399 


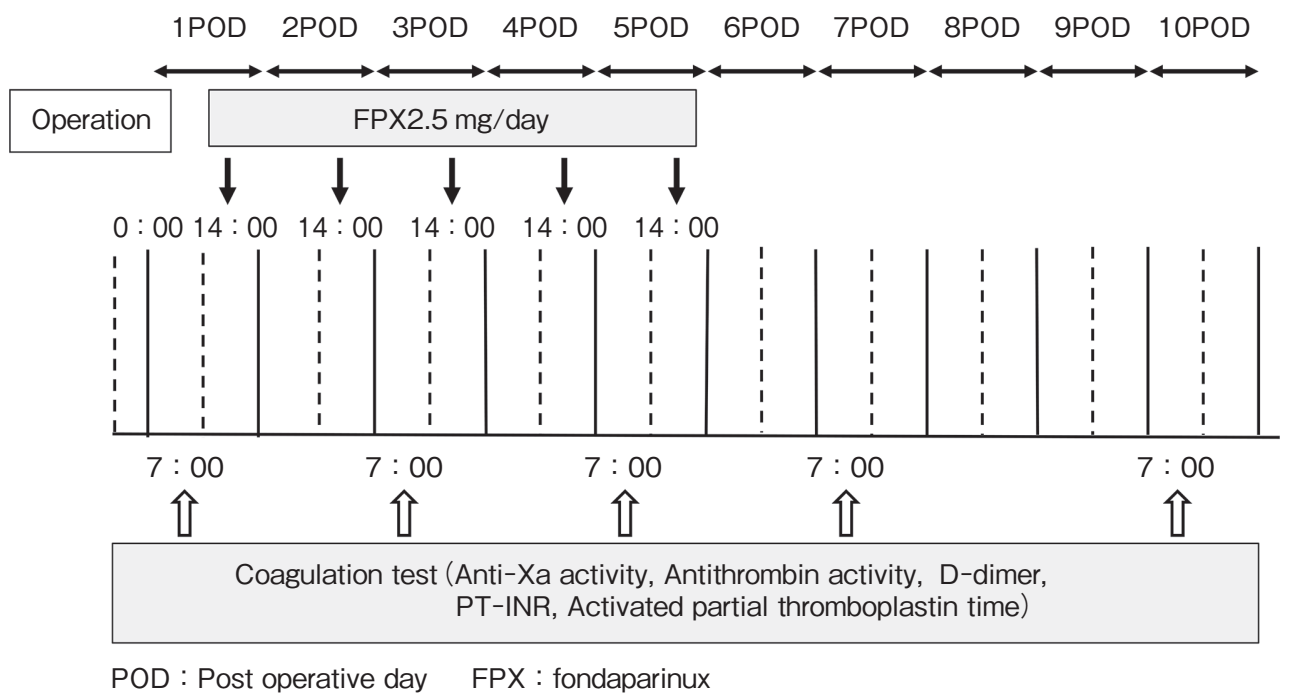

Figure-1 Study protocol

monitored using the conventional coagulation function test, Therefore prediction of both the positive effect and adverse events was considered difficult. However, monitoring based on inhibitory activity of activated blood coagulation factor $\mathrm{X}$ (anti-Xa activity) was studied in the orthopedic field, and this monitoring method provided good results in some studies, but the findings were not consistent ${ }^{6 \text { ) }}{ }^{7)}$.

In this study, we monitored anti-Xa activity over time during postoperative anticoagulant therapy with FPX after abdominal surgery and investigated the usefulness of this method.

\section{Subjects and Methods}

\section{Subjects}

The subjects were 67 patients who underwent resection of a primary colorectal cancer lesion under a combination of general and epidural anesthesia between December 2010 and October 2011 at Juntendo University Hospital, Tokyo, Japan. Patients with multiple tumors and double cancer and patients treated with palliative surgery, such as bypass surgery and colostomy, were excluded. Patients weighing $30 \mathrm{~kg}$ or lower, those with renal dysfunction with an estimated glomerular filtration rate (eGFR) of below $30 \mathrm{ml} / \mathrm{min} / 1.73 \mathrm{~m}^{2}$, and those treated with either antiplatelet or anticoagulant therapy before surgery were also excluded.

For staging of colorectal cancer, TNM Classification of Malignant Tumours (7th edition) ${ }^{12)}$ was used. Regions containing colorectal cancer were classified into 2 groups: right side (cecum, ascending colon, and transverse colon) and left side (descending colon, sigmoid colon, and rectum).

\section{VTE prevention method}

Intermittent pneumatic compression (IPC) was applied as a physical therapy to prevent VTE in all patients. This was initiated during surgery and continued until the patient was able to walk. For drug therapy, $2.5 \mathrm{mg}$ /day of FPX was subcutaneously administered at 14:00 daily from postoperative day 1 to 5 (Figure-1). The epidural catheter was removed at 10:00 on day 2 after confirming the absence of bleeding.

\section{Diagnosis of adverse events}

Deep vein thrombosis (DVT) and pulmonary embolism (PE) were diagnosed based on clinical symptoms. Regarding bleeding adverse events, patients with Clavien-Dindoclassified ${ }^{13)}$ Grade I or more severe bleeding and without bleeding were classified as the bleeding and non-bleeding groups, respectively. When a bleeding occurred, subsequent FPX administration was discontinued unless hemostasis could not be confirmed.

\section{Parameters measured and methods}

The parameters that were measured included the anti-Xa activity, Antithrombin activity, D-dimer, Prothrombin time-international normalized ratio (PT-INR), and Activated partial thromboplastin time (Figure-1). Coagulation tests were performed at 7:00 on postoperative days $1,3,5$, and 7 . It was 
Table-1 Patients' characteristics

\begin{tabular}{|c|c|c|c|}
\hline & Bleeding group $(\mathrm{n}=11)$ & Non-Bleeding group $(n=56)$ & p-value \\
\hline Age (year) & $64(41-75)$ & $62(34-83)$ & 0.493 \\
\hline Gender (male : female) & $8: 3$ & $27: 29$ & 0.137 \\
\hline Weight $(\mathrm{kg})$ & $61.2(43.3-77.4)$ & $61.0(36.1-101.5)$ & 0.933 \\
\hline BMI $\left(\mathrm{kg} / \mathrm{m}^{2}\right)$ & $22.8(17.7-27.7)$ & $23.0(14.8-40.5)$ & 0.606 \\
\hline $\mathrm{eGFR}\left(\mathrm{m} l / \mathrm{min} / 1.73 \mathrm{~m}^{2}\right)$ & $83.1(30.1-107.2)$ & $80.1(46.1-117.4)$ & 0.735 \\
\hline Stage $^{*}$ Stage 0 & 0 & 2 & \\
\hline Stage I & 3 & 21 & \\
\hline Stage II & 3 & 15 & 0.831 \\
\hline Stage IIIa, b & 4 & 16 & \\
\hline Stage IV & 1 & 2 & \\
\hline Tumor site (Right : Left) & $7: 4$ & $17: 39$ & 0.035 \\
\hline Operating time $(\min )$ & $249(159-371)$ & $235(109-507)$ & 0.729 \\
\hline Intraoperative blood loss $(\mathrm{g})$ & $20(10-230)$ & $25(6-460)$ & 0.670 \\
\hline Operative method (Open : LAP) & $1: 10$ & $13: 43$ & 0.292 \\
\hline $\begin{array}{l}\text { Anastomotic type } \\
\text { (None: Hand-sewn : FEEA : DST) }\end{array}$ & $0: 0: 6: 5$ & $3: 3: 18: 32$ & 0.441 \\
\hline Postoperative hospital stay (day) & $12(9-24)$ & $11(8-65)$ & 0.527 \\
\hline
\end{tabular}

*Stage: TNM Classification of Malignant Tumours (7th edition)

BMI: Body Mass Index eGFR: estimated glomerular filtration rate LAP: Laparoscopic surgery

FEEA: functional end-to-end anastomosis DST: double stapling technique

also performed on day 10 in those patients who stayed in the hospital longer. A solution of $3.8 \%$ sodium citrate was used as the anticoagulant for blood sampling. After blood sampling, plasma was immediately separated and stored at $-30^{\circ} \mathrm{C}$ until analysis. The anti-Xa activity was measured by a chromogenic assay using a synthetic chromogenic substrate $\left(\mathrm{S}-2222^{\circledR}\right.$, SEKISUI MEDICAL). Antithrombin activity was measured using the Test TeamS ATIII ${ }^{\circledR}$ (SEKISUI MEDICAL). D-dimer was measured using Nanopia D-dimer ${ }^{\circledR}$ (SEKISUI MEDICAL). PT-INR was determined using Coagpia PT-N ${ }^{\circledR}$ (SEKISUI MEDICAL). The activated partial thromboplastin time was mesured using Coagpia APTT-N ${ }^{\circledR}$ (SEKISUI MEDICAL). An automatic measurement device (CorePresta2000 ${ }^{\circledR}$, SEKISUI MEDICAL) was used for these studies.

\section{Statistical analysis}

All values in the text are presented as the median. For statistical analysis, a univariate analysis was performed using the Mann-Whitney-U test, Fisher's exact test, and $\chi^{2}$ test. Items in which a significant difference was detected were subjected to multivariate analysis using logistic regression analysis. The analytical results were judged as significant when the $\mathrm{p}^{-}$-value was $<0.05$. In the bleeding group, the anti-Xa activity level was investigated in patients who had a bleeding that occurred after each measurement day. To predict the development of bleeding based on the anti-Xa activity level, a receiver operating characteristic curve was prepared from the anti-Xa activity level, the cut-off value was set using the Youden index, and the area under the curve (AUC) was calculated.

This study was performed with approval by the Institutional Review Board of Juntendo University Hospital (approval number: 546) and with written consent from all patients.

\section{Results}

The median age of all 67 patients was 63 years old (34-83 years old). There were 35 male and 32 female patients with a median weight $61.0 \mathrm{~kg}$ $(36.1-101.5 \mathrm{~kg})$. The median eGFR was $80.5 \mathrm{ml} /$ $\min / 1.73 \mathrm{~m}^{2}\left(46.1-117.4 \mathrm{~m} l / \mathrm{min} / 1.73 \mathrm{~m}^{2}\right)$. No symptomatic DVT or PE developed in any patient. Bleeding was noted in 11 patients (16.4\%).

The background factors of the bleeding (11 patients) and the non-bleeding (56 patients) groups are shown in Table-1. No significance 
Table-2 Bleeding event and treatment in the bleeding group

\begin{tabular}{|c|c|c|c|c|c|c|c|}
\hline Case & Bleeding events & $\begin{array}{l}\text { Tumor site } \\
\text { (Right : Left) }\end{array}$ & $\begin{array}{l}\text { Onset of bleeding } \\
\text { (POD) }\end{array}$ & Grade* $^{*}$ & Treatment & $\begin{array}{l}\text { Anastomosis } \\
\text { method }\end{array}$ & $\begin{array}{c}\text { Most recent } \\
\text { anti-Xa activity level } \\
(\mu \mathrm{g} / \mathrm{m} l)\end{array}$ \\
\hline 1 & $\begin{array}{l}\text { Bleeding from site of } \\
\text { drainage tube }\end{array}$ & $\mathrm{L}$ & 2 & I & Astriction & DST & 0 \\
\hline 2 & $\begin{array}{l}\text { Bleeding from site of } \\
\text { drainage tube }\end{array}$ & $\mathrm{L}$ & 3 & I & Astriction & DST & 0.25 \\
\hline 3 & $\begin{array}{l}\text { Bleeding from the } \\
\text { surgical wounds }\end{array}$ & $\mathrm{R}$ & 3 & I & Astriction & FEEA & 0.28 \\
\hline 4 & $\begin{array}{l}\text { Gastrointestinal } \\
\text { bleeding }\end{array}$ & $\mathrm{R}$ & 4 & I & $\begin{array}{c}\text { Discontinuation of } \\
\text { FPX }\end{array}$ & FEEA & 0.16 \\
\hline 5 & $\begin{array}{l}\text { Gastrointestinal } \\
\text { bleeding }\end{array}$ & $\mathrm{R}$ & 4 & I & $\begin{array}{c}\text { Discontinuation of } \\
\text { FPX }\end{array}$ & FEEA & 0.18 \\
\hline 6 & $\begin{array}{l}\text { Gastrointestinal } \\
\text { bleeding }\end{array}$ & $\mathrm{R}$ & 4 & I & $\begin{array}{c}\text { Discontinuation of } \\
\text { FPX }\end{array}$ & FEEA & 0.19 \\
\hline 7 & $\begin{array}{l}\text { Gastrointestinal } \\
\text { bleeding }\end{array}$ & $\mathrm{R}$ & 4 & I & $\begin{array}{c}\text { Discontinuation of } \\
\text { FPX }\end{array}$ & FEEA & 0.17 \\
\hline 8 & $\begin{array}{l}\text { Atypical genital } \\
\text { bleeding }\end{array}$ & $\mathrm{L}$ & 5 & I & $\begin{array}{c}\text { Discontinuation of } \\
\text { FPX }\end{array}$ & DST & 0.23 \\
\hline 9 & $\begin{array}{l}\text { Acute subdural } \\
\text { hematoma }\end{array}$ & $\mathrm{R}$ & 5 & II & $\begin{array}{c}\text { Administration of } \\
\text { hemostat }\end{array}$ & FEEA & 0.32 \\
\hline 10 & $\begin{array}{l}\text { Bleeding from site of } \\
\text { drainage tube }\end{array}$ & $\mathrm{R}$ & 6 & IIIa & Suture hemostasis & DST & 0.10 \\
\hline 11 & $\begin{array}{l}\text { Gastrointestinal } \\
\text { bleeding }\end{array}$ & $\mathrm{L}$ & 1 & IIIa & $\begin{array}{l}\text { Endoscopic } \\
\text { hemostasis }\end{array}$ & DST & 0.01 \\
\hline
\end{tabular}

* Grade: Clavien-Dindo classification

POD: Post operative day DST: Double stapling technique FEEA: Functional end-to-end anastomosis FPX: fondaparinux

difference was noted in patient factors other than the location of the colorectal cancer between the 2 groups. The location of the colorectal cancer was on the right side in many cases in the bleeding group. No significance difference was noted in surgical factors (surgical time, blood loss during surgery, surgical procedure, or gastrointestinal anastomosis method) between the 2 groups, nor was there a significance difference in the duration of the postsurgical hospital stay.

The details of the bleeding group (11 patients) are shown in Table-2. Gastrointestinal bleeding occurred most frequently (5 patients, 7.5\%), followed by bleeding at the drain insertion site in 3 patients (4.5\%). In the 5 patients who had a gastrointestinal bleeding, the gastrointestinal anastomosis method was a functional end-to-end anastomosis (FEEA) in 4 patients and a double stapling technique (DST) in 1 patient. Epidural catheter-related bleeding was not observed. Serious grade IIIa bleeding was noted in 2 patients, and the types were gastrointestinal bleeding and bleeding at the drain insertion site. For the gastrointestinal bleeding patients, endoscopic hemostasis was applied to the bleeding at the anastomosis site on day 1 . For patients with a drain insertion site bleeding, a hemostatic suture was performed on day 6 .

Changes in the anti-Xa activity are shown in Figure-2. There were 11 patients in the bleeding group in whom the anti-Xa activity was investigated but bleeding also occurred in 2 patients with bleeding before day 3 . Thus, we studied 9 of the bleeding patients excluding these 2 patients. Similarly, three patients were studied who had a bleeding on day was 5 . Since bleeding occurred earlier than day 7 in all patients, the anti-Xa activity was not investigated in any patient after day 7 . The anti-Xa activity gradually rose after initiation of administration in both the bleeding and non-bleeding groups until day 5 and then the anti-Xa activity in the non-bleeding group rapidly decreased thereafter. The bleeding group has not the date on day 7 and 10. Therefore the line is stopped on day 7 . When the anti-Xa activity was compared between the bleeding and non-bleeding groups, the 


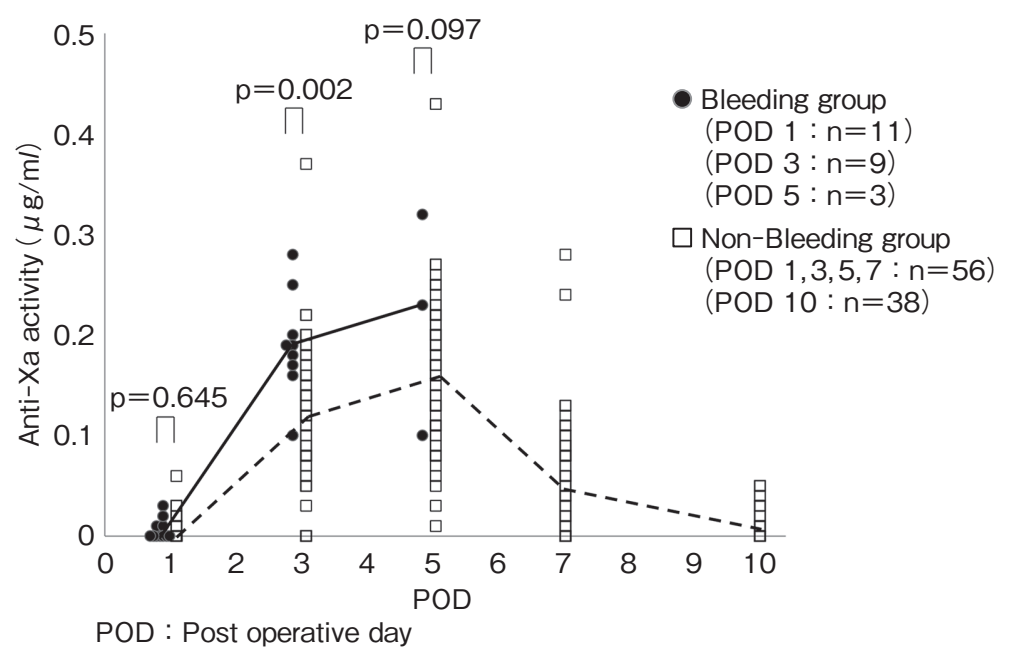

Figure-2 Changes in anti-Xa activity level

Table-3 Changes in clotting functions other than the anti-Xa activity level

\begin{tabular}{lccccc}
\hline & $\begin{array}{c}\text { POD1 } \\
(\mathrm{n}=67)\end{array}$ & $\begin{array}{c}\text { POD3 } \\
(\mathrm{n}=65)\end{array}$ & $\begin{array}{c}\text { POD5 } \\
(\mathrm{n}=59)\end{array}$ & $\begin{array}{c}\text { POD7 } \\
(\mathrm{n}=56)\end{array}$ & $\begin{array}{l}\text { POD10 } \\
(\mathrm{n}=38)\end{array}$ \\
\hline $\begin{array}{l}\text { Antithrombin activity } \\
(70-130 \%) *\end{array}$ & 83.9 & 89.6 & 95.8 & 99.8 & 101.9 \\
$\begin{array}{l}\mathrm{D}-\text { dimer } \\
(\leqq 1.0 \mu \mathrm{g} / \mathrm{m} l)\end{array}$ & 3.3 & 2.0 & 2.3 & 2.5 & 2.3 \\
$\begin{array}{l}\text { PT-INR }(1.0) \\
\text { Activated partial } \\
\text { thromboplastin time } \\
(24-39 \text { s) }\end{array}$ & 1.18 & 1.15 & 1.16 & 1.15 & 1.13 \\
\hline $\begin{array}{l}\text { ( }) * \text { Normal value } \\
\text { POD: Post operative day }\end{array}$ & 35.3 & 39.2 & 35.6 & 33.7 & 32.3 \\
\end{tabular}

Table-4 Multivariate analysis of cases with bleeding

\begin{tabular}{lccc}
\hline & $\mathrm{p}$-value & Odds ratio & 95\% Confidence interval \\
\hline Anti-Xa activity (POD3) & 0.021 & 5.37 & $3.83-30.43$ \\
Tumor site (Right:Left) & 0.051 & 5.44 & $0.99-43.64$ \\
\hline
\end{tabular}

POD: Post operative day

anti-Xa activity was higher in the bleeding group on day $3(0.19 \mu \mathrm{g} / \mathrm{m} l$ vs. $0.12 \mu \mathrm{g} / \mathrm{m} l, \mathrm{p}=0.002)$.

No significant variation was noted in the coagulation parameters (Antithrombin activity, D-dimer, PT-INR, or Activated partial thromboplastin time) other than that of the anti-Xa activity throughout the study (Table-3). In addition, the coagulation parameters other than the anti-Xa activity were compared between the bleeding and non-bleeding groups, but no significant difference was noted in any parameter.

Significant differences between the bleeding and non-bleeding groups using univariate analysis were noted in the anti-Xa activity level on day 3 and the location of the colorectal cancer lesion. When logistic regression analysis was performed regarding these as explanatory variables, only the anti-Xa activity on day 3 was a significant factor (odds ratio: $5.37, \mathrm{p}=0.021)($ Table -4$)$.

When the cut-off value of the anti-Xa activity level on day 3 was set at $0.16 \mu \mathrm{g} / \mathrm{ml}$, the AUC when a bleeding was found was 0.806 . The anti-Xa activity level on day 3 exceeded the cut-off value in 23 patients, and bleeding was noted in 8 (34.8\%) of the patients. 


\section{Discussion}

Clinical studies on the VTE-preventive effect of FPX in the abdominal surgery have been reported by the APOLLO trial performed overseas ${ }^{14)}$ and a randomized study in Japan performed by Sakon et al. ${ }^{15)}$. In both studies, the efficacy of $2.5 \mathrm{mg} /$ day of FPX was investigated, and a reduction of the incidence of VTE was observed.

The incidence of bleeding was higher than that in the placebo group ( $2.4 \%$ vs. $0.7 \%$ ) in the APOLLO trial, and the incidence was also high ( $2.6 \%$ vs. $0 \%)$ in the study performed by Sakon et al. In our study, bleedig was noted in 11 (16.4\%) of the 67 patients treated with FPX. The incidence of bleeding in the Sakon $e$ al. study performed in Japan was $2.6 \%$, and was lower than that (16.4\%) in our study, and the severity of bleeding was also mild (grade I). One reason for this difference in incidence may have been the delayed initiation of FPX administration in their study compared with that in our study. During this study period, 111 patients who underwent surgery for colorectal cancer at Juntendo University Hospital were treated only with IPC without anticoagulant therapy, bleeding was noted in $3(2.7 \%)$ of them, and it was gastrointestinal bleeding in one $(0.9 \%)$. Compared with these patients treated only with IPC, the incidence of bleeding increased by 6 -fold in our study, suggesting that anticoagulant therapy with FPX increased the number of bleeding patients. Physicians should be aware of this problem. In other reports from the abdominal surgery, the incidence of bleeding varied from $0 \%$ to $38.4 \%{ }^{15)-17)}$, suggesting that the daily dose, timing of initiation of administration, and differences in the definition and evaluation method for bleeding influence the incidence. We observed the significant difference of the location of the colorectal cancer in the bleeding group (right side $>$ left side) for reasons that are unknown and required further investigation.

In our study, gastrointestinal bleeding occurred (5 patients, $7.5 \%$ ) most frequently. The incidence of gastrointestinal bleeding after surgery for colorectal cancer was reported to be $2.2-3.8 \%{ }^{18)}{ }^{19}$, suggesting that anticoagulant therapy with FPX elevated the incidence of gastrointestinal bleeding. For gastrointestinal bleeding early after surgery for colorectal cancer, endoscopic hemostasis is not always the first-choice for all patients because the site and method of anastomosis vary ${ }^{18)}$. In our study, bleeding on the right side of the colon (4 patients) responded to conservative treatment, including discontinuation of FPX. In these 4 patients, FEEA was applied employing the semi-closed method in which an automatic suture instrument was used twice. Hemostasis was ensured in a semi-closed state after the first use of the automatic suture instrument, but gastrointestinal bleeding could not be prevented. In a rectal cancer patient in whom grade IIIa gastrointestinal bleeding occurred, hemostasis at the anastomosis was not endoscopically confirmed during surgery. Endoscopy during surgery using an automatic anastomosis device was effective as a countermeasure for bleeding at the anastomosis in a previous study ${ }^{20)}$, suggesting its use should be investigated for patients who will receive anticoagulant therapy after surgery. Acute subdural hematoma was noted in one patient $(1.5 \%)$. When anticoagulant therapy is performed, intracranial bleeding should also be taken into consideration as an adverse event ${ }^{111}$.

Anti-Xa activity was useful in predicting bleeding in the orthopedic field in some reports ${ }^{7)}$ but it was not useful in other field ${ }^{6)}$, and no consensus viewpoint has been reached. It has been reported that the terminal half-life of fondaparinux was 17 hours in young volunteers and 21 hours in elderly volunteers ${ }^{21)}$. In our study, the activity was high in the bleeding group when it was measured near the trough level on day 3, and thus it may serve as a bleeding-predictive factor in the presence of FPX administration in the abdominal surgery. It has been reported that continuation of FPX administration gradually increases anti-Xa activity ${ }^{62}{ }^{22}$, and it also gradually increased in our study. We suggest that development of a bleeding can be predicted by measuring anti-Xa activity on day 3 during anticoagulant therapy with FPX, and information of this activity can help to decide whether to temporarily discontinue or reduce the dose of anticoagulant therapy. Since the timing of anti-Xa activity measurement was set at postsurgical days $1,3,5,7$, and 10 , bleeding on postoperative days 1 and 2 could not be predicted, it may be possible to predict bleeding after day 2 by measuring the activity on day 2 . Among the time-points of anti-Xa activity measurement, day 3 was judged as the 
appropriate time-point because many cases of bleeding could be predicted. Since the incidence of bleeding was higher than that expected in anticoagulant therapy with FPX for prevention of postoperative VTE, attention should be paid to the possibility of bleeding when this therapy is used. In our study, the AUC value of anti-Xa activity level on day 3 was more than 0.8 , and we suggest that the anti-Xa activity serves as an index to predict occurrence of bleeding. By monitoring the anti-Xa activity measured value at which VTE is prevented without causing bleeding, safe anticoagulant therapy with FPX without bleeding and preventing VTE may be performed.

\section{Acknowledgment}

We are grateful to the staff of the Clinical Laboratory Department of Juntendo University Hospital for their cooperation in performing the coagulation-related tests in this study.

\section{Conflict of interests}

The authors declare no conflict of interest associated with this manuscript.

\section{References}

1) Editorial Committee on Japanese Guideline for Prevention of Venous Thromboembolism. Japanese Guideline for Prevention of Thromboembolism. Tokyo: Medical Front International Limited, 2004.

2) Sakon M, Ikeda M: Venous thromboembolism in abdominal cancer surgery. Clin J Gastroenterol, 2009; 2: 247251.

3) Suzuki I, Ozeki Y: Preclinical and clinical data of the synthetic Xa inhibitor fondaparinux. Folia Pharmacologica Japonica, 2012; 139: 117-126.

4) Montagnac R, Brahimi S, Janian P, Melin JP, Bertocchio JP, Wynckel A: Use of fondaparinux during hemodialysis in heparin-induced thrombocytopenia. About a new observation. Nephrol Ther, 2010; 6: 581-584.

5) Rupprecht HJ, Blank R: Clinical pharmacology of direct and indirect factor Xa inhibitors. Drugs, 2010; 70: 21532170.

6) Yoshida K, Wada H, Hasegawa $M$, et al: Increased fibrinolysis increases bleeding inorthopedic patients receiving prophylactic fondaparinux. Int J Hematol, 2012; 95: 160-166.

7) Wada H, Shimokariya Y, Sakaguchi A: Monitoring of anti-Xa activity in orthopedic patients receiving prophylactic anti-thrombotic therapy. Japanese Journal of Clinical Laboratory Automation, 2013; 38: 184-186.

8) Yazawa T, Takenami T, Ono T, et al: Optimal strategy for the diagnosis of deep Vein thrombosis and effects of fondaparinux used in thromboembolism prophylaxis in gastric cancer surgery. J Abdom Emerg Med, 2013; 33: 1131-1136.

9) Matinez L, Burnett A, Borrego M, Streeter JC, Townsend K, Garcia D: Effect of fondaparinux prophylaxis on anti-factorXa concentrations in patients with morbid obesity. Am J Health Syst Pharm, 2011; 68: 1716-1722.

10) Iba $T$, Aihara $K$, Watanabe $S$, et al: Factor Xa inhibitor attenuates leukocyte adehesion and thrombous formation in an experimental mouse model of the metabolic syndrome. Cardiovasc Ther, 2013; 31: 280-284.

11) Manabe T, Ohdera T, Katsuki M: Bleeding complications in anticoagulant treatment using fondaparinux after total knee arthroplasty. Orthopedics \& Traumatology, 2010; 59: 216-219.

12) Union for International Cancer Control: TNM Classification of Malignant Tumours 7th edition. In: Sobin LH, Gospodarowicz MK, Wittekind C, eds. Geneva: Union for International Cancer Control, 2009.

13) Dindo D, Demartines N, Clavien PA: Classification of surgical complications: a new proposal with evaluation in a cohort of 6336 patients and results of a survey. Ann Surg, 2004; 240: 205-213.

14) Turpie AG, Bauer KA, Caprini JA, Comp PC, Gent M, Muntz JE; Apollo Investigators: Fondaparinux combined with intermittent pneumatic compression vs. intermittent pneumatic compression alone for prevention of venous thromboembolism after abdominal surgery: a randomized, double-blind comparison. J Thromb Haemost, 2007; 5: 1854-1961.

15) Sakon M, Tukamoto T, Kobayashi T, Fujita S, Kawashima T, Monden M: Clinical evaluation of fondaparinux for prevention of venous thromboembolism after abdominal surgery: a randomized open-label study of fondaparinux and intermittent pneumatic compression as a benchmark. Clin J Therap Med, 2008; 24: 679-689.

16) Yamaguchi H, Furuhata T, Okita K, et al: Prophylaxis against venous thromboembolism after laparoscopic colorectal cancer surgery: safety of our protocol using fondaparinux. J Abdom Emerg Med, 2013; 33: 1137-1143.

17) Hayashi H, Morikawa T, Motoi F, et al: Evaluation of the safety and efficacy of postoperative thromboprophylaxis after hepatobiliary-pancreatic surgery. J Abdom Emerg Med, 2013; 33: 1157-1164.

18) Masuzawa T, Tominaga S, Miyagaki H, Fukuzaki T: A successfully treated case by APC for postoperative bleeding of FEEA. J JPN Soc Coloprotol, 2008; 61: 329332.

19) Tagawa T, Oota $Y$, Nakano $Y$, Nagayasu T, Adachi A: A clinical study of anastomosis in the reconstruction for anterior resection of the rectum and sigmoidectomy: an efficacy of double stapling technique. The Japanese Journal of Gastroenterological Surgery, 1992; 25: 27732778.

20) Enomoto T, Saida Y, Nakamura Y, et al: Intraoperative colonoscopy of anterior resection for rectal cancer. Progress of Digestive Endoscopy, 2007; 71: 39-41.

21) Donat F, Duret JP, Santoni A, et al: The pharmacokinetics of fondaparinux sodium in healthy volunteers. Clin Pharmacokinet, 2002; 41 Suppl 2: 1-9.

22) Kudrnová Z, Kvasnicka J, Kudarna K, et al: Favorable coagulation profile with fondaparinux after hip surgery in elderly patients. Int J Hematol, 2009; 90: 476-482. 